\title{
Muscular dystrophy meets the mesangioblast
}

A recently discovered population of stem cells, dubbed mesangioblasts, can repair muscle tissue and improve motor ability in a mouse model of limbgirdle muscular dystrophy.

Sampaolesi et al. found that mesangioblasts injected into the mice, which were deficient in the $\alpha$-sarcoglycan gene, restored expression of $\alpha$ sarcoglycan and improved muscle fiber morphology several months after treatment. In the 10 July Science they also report that the treated animals were able to walk on a rotating wheel for longer than untreated animals, although not as long as healthy mice. The authors obtained similar results with $\alpha$-sarcoglycan deficient mesangioblasts corrected with the $\alpha$ sarcoglycan gene and expanded in culture (GFP- $\alpha$-sarcoglycan shown here in treated area after 4 months, untreated, contralateral area in insert).

Mesangioblasts are a class of vessel-associated stem cells that can differentiate into most mesodermal tissues. In mice they are found in the fetus, and, at much lower frequency, in adults. The authors speculate that

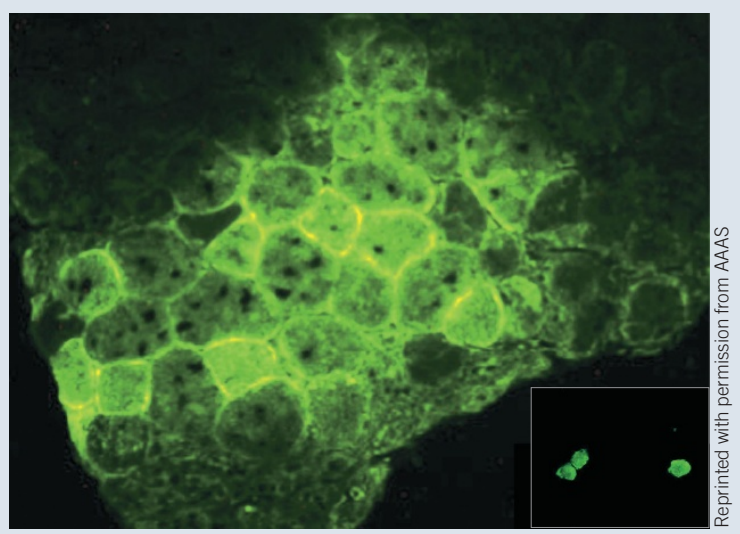
mesangioblasts might also hold promise for other types of dystrophies especially in combination with techniques of gene repair. Methods such as exon skipping, described by Lu et al. (pages 1009-1014) and Tidball and Spencer (pages 997-998) could potentially be used in culture to correct the primary defect in mesangioblasts prior to injection.

Charlotte Schubert

\section{Road map to metastasis}

\section{Laura J Van't Veer \& Britta Weigelt}

\section{When do cancer cells acquire metastatic identity? Recent reports have indicated that the propensity to metastasize might be hardwired early during tumor development. Now it seems that breast cancer cells might acquire a gene signature early in tumorigenesis that predicts the site of metastasis.}

The occurrence of metastases in distant organs is the major cause of death for cancer patients. At the time of diagnosis of the primary tumor, undetectable, disseminated micrometastases may already be present; these ultimately kill half of all cancer patients, depending on the tumor type (metastases kill the large majority of lung cancer patients, and about a third of those with breast cancer).

In a recent issue of Cancer Cell, Kang et al. ${ }^{1}$ identify, in a human breast cancer cell line, a specific set of genes that mediates metastasis to bone when these cells are injected into the cardiac ventricle of immunodeficient mice. Tumor cells isolated from these bone lesions, after a second round of injection, gave rise to two subpopulations of cells: one with elevated bonemetastatic potential compared with the parental cells, and another with low bonemetastatic potential but high activity toward the adrenal glands.

Laura J. Van't Veer and Britta Weigelt are at The Netherlands Cancer Institute, Molecular Pathology, 121 Plesmanlaan, 1066 cx Amsterdam, The Netherlands.

e-mail: I.vt.veer@nki.nl or b.weigelt@nki.nl
The authors performed microarray analysis on the two subpopulations and found that expression of 102 genes can distinguish between strong or weak bone-metastasis potential. Furthermore, the parental MDAMB-231 cells and all derivative subpopulations displayed the 'poor-prognosis' gene expression signature of 70 genes, which was recently identified in primary human breast tumors as being indicative for high metastatic potential and poor patient survival ${ }^{2,3}$.

Kang et al. functionally validated four of the most highly overexpressed genes from the panel of 102 bone-metastasis genes. All four genes take part in the osteolytic pathway: interleukin (IL)11 , connective tissue growth factor (CTGF), chemokine (C-X-C motif) receptor-4 (CXCR-4) and matrix metalloproteinase-1 (MMP-1). Transfected MDA-MB-231 cells overexpressing any two of the four genes in combination with osteopontin (OPN), a secretory protein that promotes osteoclast function, showed a highly increased incidence of bone metastases in mice comparable to that of the in vivo selected subpopulations with high bone-metastatic activity.

The authors next asked whether the subpopulations of elevated metastatic potential already reside in the parental MDA-MB-231 cell line. They evaluated expanded single-cell cultures for metastatic activity and expression of IL-11, MMP-1, CTGF, CXCR-4 and OPN. Cell populations expressing four or five of the selected genes were highly metastatic in vivo, whereas populations expressing none of those genes generated no bone metastases.

According to the prevailing model of metastasis, the primary tumor is biologically heterogeneous, and metastatic capacity is acquired only rarely and late in tumorigenesis ${ }^{4,5}$ (Fig. 1a). Only a minority of tumor cells from subpopulations with advantageous potential has the ability to escape from the primary tumor mass and form metastases at distant sites.

Recently, it has been postulated that the capacity to metastasize might be acquired relatively early during multistep tumorigenesis and is intrinsic to the tumor ${ }^{2,6}$ (Fig. 1b). This hypothesis is based on findings that in human breast cancer, expression profiles of the whole primary tumor can predict disease outcome of breast cancer patients ${ }^{2,3,7}$. A poor-prognosis signature is strongly predictive for the development of distant metastases, in contrast to the 'good-prognosis' signature. 